Fictionalised case presentations (sometimes including dialogue between therapist and patient) are used throughout to illustrate concepts and the practicalities of modified CBT.

This book is not written for psychiatrists, clinical psychologists or mental health nurse therapists. The stated aim is to encourage clinicians within general healthcare settings to provide CBTinformed interventions to patients with health anxiety. While accepting the arguments for this approach, it is uncertain that clinicians untrained in CBT would find the book sufficiently detailed to successfully deliver modified CBT; failure to mention clinical supervision of therapists is surprising. The explicit rejection of psychoanalytic mechanisms as having any role in the aetiology of health anxiety may be undermined by clinical examples that appear to show symptom resolution after 'catharsis. It was disappointing that the concept of pathologically low anxiety about one's health (e.g. in people engaging in high-risk behaviours), mentioned in the introduction, is not developed. Little evidence is presented to support the clinical efficacy of the intervention or its tolerability to patients. A few errors of editing and proof-reading persist.

Nevertheless, there is much to recommend here. The patient group will be familiar to most psychiatrists, clinical psychologists and psychiatric nurses. The writing is clear, readable and memorable with suitably detailed and easily generalised examples. The useful descriptions of therapeutic strategies make them widely and immediately applicable; pitfalls or sticking points in therapy are anticipated, with strategies for tackling them clearly laid out. Various patients I have seen were brought vividly to mind while reading and I am hopeful that my approach to similar individuals in the future will be positively enhanced by this book and more efficacious.

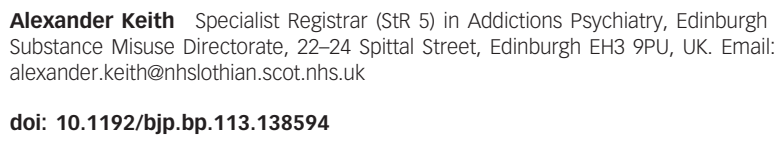

doi: 10.1192/bjp.bp.113.138594

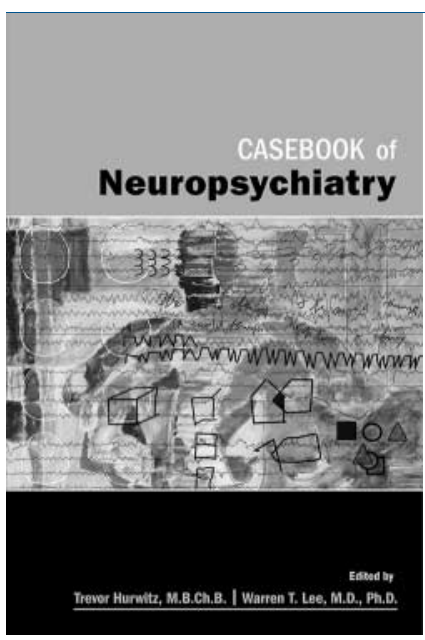

\section{Casebook of Neuropsychiatry}

Edited by Trevor Hurwitz \& Warren T. Lee. American Psychiatric Publishing 2013.

$\$ 75.00(\mathrm{pb}) .383 \mathrm{pp}$.

ISBN: 9781585624317

The discipline of clinical neuropsychiatry deals with a wide range of conditions across the domains of neurology and psychiatry. The study of organic disturbances in the central nervous system that give rise to mental diseases is equally challenging and fascinating, as illustrated by a number of recently published textbooks which have chartered this heterogeneous territory. What was missing from this rapidly evolving field was a practical tool to bridge the gap between the theory of brain-mind correlations and the practice of cases routinely seen in neuropsychiatry clinics. This casebook achieves this goal by complementing existing textbooks and bringing the subject to life through a gallery of beautifully described clinical cases.

A team of experienced neuropsychiatrists from North America have joined efforts to cover the broad spectrum of neuropsychiatric conditions, in 38 clinical cases grouped into 11 sections according to the presenting symptoms. For example, the section on hyperkinetic states features Tourette syndrome in adults, psychosis associated with Huntington's disease, and tardive dyskinesia, while the section on alterations in consciousness includes descriptions of patients with Hashimoto's encephalopathy, dissociative disorders, anti-NMDA-receptor encephalitis, neuroleptic malignant syndrome, and neuropsychiatric systemic lupus erythematosus. For each clinical presentation, the reader is provided with evidence-based information on the differential diagnosis and diagnostic workup, neurobiological aspects and current treatment options. Key clinical points, suggestions for further readings and up-to-date references are a useful addition.

This book is at the same time informative and engaging, especially for those who are susceptible to the fascination of brain-mind interaction. Each of the 11 sections opens with a short introduction accompanied by a picture of the human brain, highlighting the neuroanatomical region which is critical for the understanding of the neurobiological context of the clinical presentations. Sometimes the choice of the brain region is obvious (e.g. hippocampus and inferior temporal lobe for the section on memory failure), but at other times it can be argued that the choice is less justifiable (e.g. basal ganglia for the section on depression). These are minor shortcomings for a practical volume which epitomises the current renaissance of clinical neuropsychiatry by reviving the tradition of Charcot's Tuesday Lessons.

Andrea E. Cavanna Consultant in Behavioural Neurology, Department of Neuropsychiatry, Birmingham and Solihull Mental Health NHS Foundation Trust, and University of Birmingham; Honorary Professor in Neuropsychiatry, School of Life and Health Sciences, Aston University, Birmingham. Department of Neuropsychiatry, The Barberry National Centre for Mental Health, 25 Vincent Drive, Birmingham B15 2FG, UK. Email: A.Cavanna@ion.ucl.ac.uk

doi: 10.1192/bjp.bp.113.138347

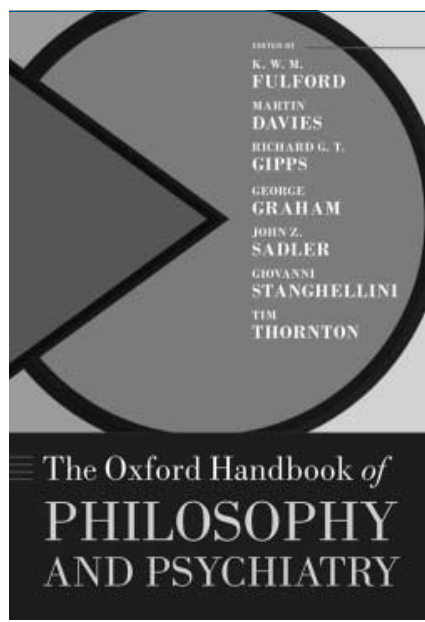

\section{The Oxford Handbook of Philosophy and Psychiatry}

Edited by K. W. M. Fulford, M. Davies, Richard G.T. Gipps, George Graham, John Z. Sadler, Giovanni Stanghellini \& Tim Thornton. Oxford University Press. 2013. £95.00 (hb). $1344 \mathrm{pp}$. ISBN: 9780199579563

Over 1300 pages long, comprising 73 chapters and featuring 90 authors, this is a massive book. Its sheer size alone is daunting, and when the editors breezily announce in the introduction that the text is primarily 'by philosophers for philosophers', one quakes a little at the prospect of actually reading it. The volume certainly 
assumes a sophisticated knowledge of philosophy on the part of the audience with much of the writing dense and technical. However, if one is selective - and the editors make the task of circumnavigating the book easier by providing short summaries at the start of each of the eight sections - there are many chapters which are not only accessible to the non-expert but also of direct relevance to clinical practice.

George Graham begins his chapter on models of madness with the story of 'the man who aimed to compose ... the Conjunction Theory of Mental Disorder' by combining every theory ever proposed. Graham uses humour to demonstrate that any attempt to erect a grand, all-encompassing explanation of psychiatric illness is doomed, because not only are competing theories often incompatible, but we have to discriminate between the plausible and the absurd. Eric Matthews draws on the work of the French philosopher Merleau-Ponty to elegantly undermine the notion that psychiatry has to choose between being either exclusively 'mindless' or 'brainless'. Matthews argues that Merlau-Ponty's contention that human beings are 'embodied subjects' helps us to see that we need both the neurosciences and psychology in psychiatry.

In their discussion of the phenomenology of delusions, Louis Sass and Elizabeth Pienkos consider Bleuler's notion of 'double bookkeeping'. Many patients seem to be able to live simultaneously in their deluded world and in the world of the sane without being unduly troubled by the apparent contradictions. Like Jaspers before them, Sass and Pienkos wonder why patients seldom follow through the implications of their delusions: for example, why is the man who believes he is God still content to sweep the hospital floor? In an attempt to understand this phenomenon, they evoke the much-used analogy of madness as a dream: in sleep, we passively give ourselves over to the absurdities of the unconscious and yet we are aware that we are dreaming.

The psychiatrist Hanna Pickard has a thoughtful chapter on personality disorder which she sees as a 'disorder of agency'. She argues that clinicians have to maintain that such patients are responsible for their actions, but, at the same time, avoid the seemingly logical step of blaming them when they falter. Denying that these patients have some degree of responsibility defies common sense, while blaming such individuals, who are often plagued by low self-worth, can be therapeutically damaging. A response by a patient, Lisa Ward, at the end of the chapter affirms that the type of approach advocated by Pickard was the one she found most helpful when she was in therapy.

Another contribution from someone who has direct experience of psychiatric problems is that by the philosopher and self-confessed alcoholic, Owen Flanagan. In one of the most interesting chapters in the book, he provides an existential perspective on addiction in which he portrays the individual as choosing the identity of the alcoholic, rather than being the passive victim of biological forces. This has consequences for treatment: it is not just a matter of the alcoholic abstaining; they have to develop a new mode of being in the world, they have to become a different person.

Towards the end of the book, Richard Askay and Jensen Farquhar compare the work of two men they consider to be the 'greatest "meta-physicians" of the twentieth century': Freud and Heidegger. These thinkers are not usually bracketed together, but the authors contend that, despite their intellectual antipathy, there are many similarities in their metaphysical approach. Both Freud and Heidegger sought to develop a comprehensive, unified account of the human condition. Both tried to uncover life's hidden meaning while accepting that there was probably not a deep, unifying meaning to human existence. Both tried to develop a new 'science of the human being' which would inform a new type of psychotherapy, in the case of Heidegger, existential analysis, in Freud's, psychoanalysis.

The editors note that their book appears exactly 100 years after psychiatry's first philosopher, Karl Jaspers published General Psychopathology. They are justified in their claim that this volume makes a major contribution to the tradition that Jaspers instigated. The involvement of practically all the major thinkers in the field and the sheer range of subjects covered makes this publication an impressive achievement.

Allan Beveridge Consultant Psychiatrist, Queen Margaret Hospital,

Whitefield Road, Dunfermline, Fife KY12 OSU, UK. Email: allanbeveridge@nhs.net

doi: 10.1192/bjp.bp.113.138248

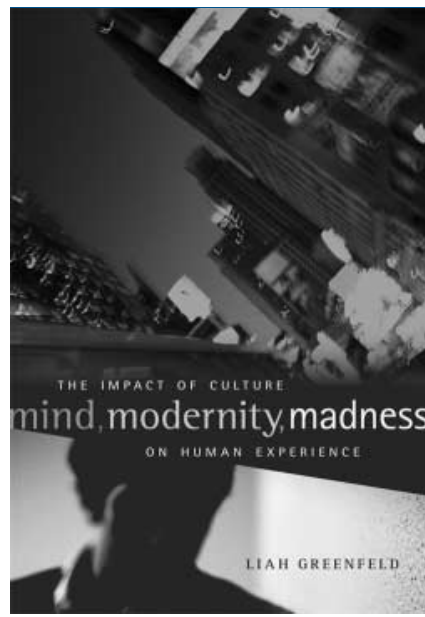

\section{Mind, Modernity, Madness: The Impact of Culture on Human Experience}

By Liah Greenfeld.

Harvard University Press. 2013.

£29.95 (hb). $688 \mathrm{pp}$.

ISBN: 9780674072763

2013 was a remarkable year for reconsideration of the nature and causes of 'madness'. In public mental health, the watershed moment came with the first report by the US Centers for Disease Control and Prevention on mental health surveillance among children: mental disorders that present before the age of 18 are becoming more common and more complex. The evolution of DSM-5 led to vigorous protests by groups such as Speak Out Against Psychiatry and interesting debates such as whether we need to change the way we are thinking about mental illness (The Observer, 12 May 2013). In his entertaining The Guardian review, titled Bipolar memoirs: What have I done? (26 April 2013), psychoanalyst Darian Leader makes a key point: 'The narrative of human lives is more or less absent in healthcare economies'.

Liah Greenfeld is a professor of sociology, political science and anthropology. Mind, Modernity, Madness completes a trilogy detailing the decline and fall of national culture. She says she was helped to find evidence on modern madness from medical libraries by her son, who 'served as [her] guide to the confused world of American young adulthood'. Since the time of Jung, anthropology has enriched our understanding of mind, and I hoped this book would add to the narrative of human lives in a way that enriched mental health policy.

Early on Greenfeld delivers her central thesis: 'A clear sense of identity being a condition sine qua non for adequate mental functioning, malformation of identity leads to mental disease, but modern culture cannot help the individual to acquire such a clear sense, it is inherently confusing' (p.5). The emergence of nationalism has been the main cause of our deforming culture, through pervasive 'anomie'. 